\title{
Regulation of Microtubule Severing by Katanin Subunits during Neuronal Development
}

\author{
Wenqian Yu, ${ }^{1 *}$ Joanna M. Solowska, ${ }^{1 *}$ Liang Qiang, ${ }^{1}$ Arzu Karabay, ${ }^{1,2}$ Douglas Baird, ${ }^{1,3}$ and Peter W. Baas ${ }^{1}$ \\ ${ }^{1}$ Department of Neurobiology and Anatomy, Drexel University College of Medicine, Philadelphia, Pennsylvania 19129, ${ }^{2}$ Department of Molecular Biology \\ and Genetics, Istanbul Technical University, 34469 Maslak-Istanbul, Turkey, and ${ }^{3}$ Department of Biology, Temple University, Philadelphia, Pennsylvania \\ 19122
}

Katanin, the microtubule-severing protein, consists of a subunit termed P60 that breaks the lattice of the microtubule and another subunit termed P80, the functions of which are not well understood. Data presented here show that the ratio of P60 to P80 varies markedly in different tissues, at different phases of development, and regionally within the neuron. P80 is more concentrated in the cell body and less variable during development, whereas P60 often shows concentrations in the distal tips of processes as well as dramatic spikes in expression at certain developmental stages. Overexpression of $\mathrm{P} 60$ at various stages in the differentiation of cultured hippocampal neurons results in substantial loss of microtubule mass and a diminution in total process length. In comparison, overexpression of P80, which is thought to augment the severing of microtubules by $\mathrm{P} 60$, results in a milder loss of microtubule mass and diminution in process length. At the developmental stage corresponding to axogenesis, overexpression of P60 decreases the total number of processes extended by the neuron, whereas overexpression of P80 produces the opposite result, suggesting that the effects on neuronal morphology are dependent on the degree of microtubule severing and loss of polymer. The microtubules that occupy the axon are notably more resistant to depolymerization in response to excess P60 or P80 than microtubules elsewhere in the neuron, suggesting that regional differences in the susceptibility of microtubules to severing proteins may be a critical factor in the generation and maintenance of neuronal polarity.

Key words: katanin; microtubule; axon; neuron; microtubule severing; neuronal polarity

\section{Introduction}

Neurons undergo dramatic morphological changes during their development, some of which can also occur in the adult. These changes involve migration of the cell body, growth of axons and dendrites, formation of axonal and dendritic branches, growth cone navigation, cessation of axonal growth, retraction of overgrown axons, formation of dendritic spines, and regeneration after injury. A growing body of evidence suggests that changes in neuronal morphology are directly related to alterations in the underlying microtubule scaffolding that contributes to the shape and architecture of various neuronal compartments (Baas and Buster, 2004). In particular, it appears that mobility of the microtubules is key to whether they can be reconfigured to accommodate changes in neuronal morphology. Recent studies have established that the length of a microtubule is inversely proportional to its capacity to move in response to forces generated by molecular motor proteins (Dent et al., 1999). In fact, the rapid movement of microtubules down the axon appears to be restricted to microtubules that are only a few micrometers in length (Wang and

\footnotetext{
Received March 2, 2005; revised April 25, 2005; accepted April 29, 2005.

This work was supported by grants from the National Institutes of Health to P.W.B. W.Y. is supported by a grant from the Edward Jekkal Foundation. We thank Sirin Korulu for assistance.

*W.Y. and J.M.S. contributed equally to this work and, therefore, should be considered first authors.

Correspondence should be addressed to Dr. Peter W. Baas, Department of Neurobiology and Anatomy, Drexel

University College of Medicine, 2900 West Queen Lane, Philadelphia, PA 19129. E-mail: Peter.W.Baas@drexel.edu. D0I:10.1523/JNEUROSCI.0834-05.2005

Copyright $\odot 2005$ Society for Neuroscience $\quad$ 0270-6474/05/255573-11\$15.00/0
}

Brown, 2002). An interesting possibility is that neurons regulate morphological changes by exercising tight control over a process called microtubule severing, in which enzymes break the lattice of the microtubule to generate multiple short microtubules from a single, long microtubule (Quarmby and Lohret, 1999). This process creates numerous short microtubules that can potentially be moved and reconfigured. Indeed, we observed microtubule severing to be particularly active at the neuronal centrosome (Yu et al., 1993), axonal branch points (Yu et al., 1994), and growth cones that begin moving after having been stalled (Dent et al., 1999).

Katanin, one of the proteins that severs microtubules, is a heterodimer composed of two subunits termed P60 and P80. The P60 subunit is the enzyme that breaks the microtubule. P80, in contrast, has no microtubule-severing properties of its own and is thought to target the P60 subunit to the centrosome (Hartman et al., 1998; McNally et al., 2000). Notably, however, both subunits are widely distributed throughout the neuron (Ahmad et al., 1999), suggesting that P80 probably has additional functions in addition to targeting to the centrosome. In the test tube, P60 severs microtubules on its own but severs even more effectively in the presence of P80 (McNally et al., 2000). An important and unresolved issue is how cells regulate the severing properties of $\mathrm{P} 60$, given that microtubules would continue to be severed into subunits if high levels of P60 were allowed unfettered access to the microtubule (McNally and Vale, 1993). One possibility is that P80 contributes to the efficacy of P60, such that alterations in the 
ratio of the two subunits could help determine the degree to which microtubules are severed. Our goals in the present study were to compare the levels and distribution of the two subunits during development of the nervous system and to perform functional tests by experimentally manipulating the two subunits in cultured fibroblasts and hippocampal neurons.

\section{Materials and Methods}

P80-katanin sequencing and preparation of P80 constructs. We previously sequenced rat P60 katanin (Karabay et al., 2004). Here, in a similar manner, we used reverse transcription-PCR to obtain the complete sequence of P80 katanin from newborn rat whole-brain tissues. Total RNA was obtained after tissue disruption with Tri Reagent (according to the instructions of the manufacturer; Sigma, St. Louis, MO) and isopropanol precipitation. cDNA was generated from total RNA using an oligo- $\mathrm{dT}_{(15)}$ primer and Powerscript reverse transcriptase (Clontech, Palo Alto, CA) according to the instructions of the manufacturer. PCR primer sequences were based primarily on available mouse and human P80 katanin sequences. Alignments identified regions of identical or highly similar nucleotide sequences that were selected as annealing sites for primers. PCR products were ligated into TA cloning vectors (Qiagen, Valencia, CA; Invitrogen, Carlsbad, CA) and then sequenced on an ABI Prism DNA Sequencer 377. Independent clones were sequenced to provide at least six readings of the cDNA of rat P80 katanin. Based on the rat P80katanin sequence, we prepared constructs for expression in cultured neurons and fibroblasts, one of which is the full-length P80-katanin sequence coupled to enhanced green fluorescent protein (EGFP) in pEGFP vector (Clontech) or myc in pCMV-Tag vector (Stratagene, La Jolla, CA). We also used the same expression vectors to clone cDNA encoding 244 C-terminal P80 amino acids to prepare the rat version of a construct termed con 80 , which has been shown to augment microtubule severing but does not target to the centrosome (McNally et al., 2000).

Preparation of anti-katanin antibodies. We previously prepared a peptide polyclonal antibody that effectively recognized P60 katanin (Karabay et al., 2004). To prepare polyclonal antibodies against P80 katanin, we cloned two fragments of P80 cDNA [P5 fragment (nucleotides 225-1150) and S3 fragment (nucleotides 1150-1968)] into the bacterial expression vector pRSET (Invitrogen). Two insoluble P80 peptides, P5 and S3, were purified from inclusion bodies using B-PER reagent (Pierce, Rockford, IL) and solubilized in $4 \mathrm{~m}$ urea. Two anti-P80 antisera, P5 and S3, were prepared by Cocalico Biologicals (Reamstown, PA) by immunizing rabbits with $\mathrm{P} 5$ or $\mathrm{S} 3$ peptide. The anti-P5 or anti-S3 IgG fractions were purified from sera by protein A-Sepharose chromatography. Antibody specificity was tested by immunolabeling and Western blot analysis of P80 transfected and nontransfected cells. We found that anti-P5 IgG is a high-titer, specific, anti-P80 antibody for immunoblotting, whereas S3 $\operatorname{IgG}$ is better suited for immunostaining. To use anti-P60 and anti-P80 antibodies for quantitative Western blot analysis, we calibrated both antibodies by immunoblotting with known amounts of purified, fulllength P60 or P80 katanin. P60 and P80 cDNAs were cloned into the pRSET bacterial expression vector (Invitrogen). P60 and P80 katanins were produced and purified from inclusion bodies using B-PER reagent and solubilized in $4 \mathrm{~m}$ urea. The concentration of full-length katanin P60 and P80 was estimated densitometrically after SDS-PAGE and staining with GelCode Blue Stain reagent (Pierce) using albumin as a standard. To obtain P60 and P80 standard curves, SDS-PAGE was performed using $20-80 \mathrm{fmol}$ of purified, full-length $\mathrm{P} 80$ or $20-160 \mathrm{fmol}$ of purified, full-length P60 katanin. After electrophoresis, proteins were transferred to nitrocellulose and immunoblotted with anti-P80 or anti-P60 antibody (diluted 1:20,000). Optical density (OD) was measured for each protein band corresponding to different concentrations of full-length P80 or P60, and standard curves were drawn.

Quantitative Western blot analysis. Rat neuronal tissues (cerebral cortex, hippocampus, cerebellum, and spinal cord) and non-neuronal tissues (heart and lungs) were isolated at different stages of development [embryonic day 13 (E13) and E18; postnatal day 0 (P0), P6, and P12; and adult]. To obtain enough tissue for accurate weighing, adjustment of total protein concentration for electrophoresis, and subsequent quanti- tative Western blot analysis, we collected and combined E15 and E18 tissues from 12 individual animals at each stage, P0 and P6 tissues from six to eight animals, and P12 and adult tissues from four to six animals. The highest number of animals was used to collect hippocampal and spinal cord tissues because of their small sizes. Tissues were collected on dry ice, weighed, and homogenized in Laemmli sample buffer using $1 \mathrm{ml}$ of buffer/100 mg of tissue. To quantify P60 and P80 levels, protein extracts from $1000 \mu \mathrm{g}$ of tissue from different developmental stages were used. One-half of the extract was used for Western blot analysis with anti-P80 antibody, and the other half was used simultaneously for Western blot analysis with anti-P60 antibody. To assure that protein loads were the same for all tissues and time points, we verified protein concentrations on gels using GelCode Blue Stain reagent (Pierce) and adjusted final dilutions if necessary.

SDS-PAGE and Western blot analysis were performed according to established procedures (Sambrook et al., 1989). Blots were blocked with $5 \%$ nonfat milk in TTBS (50 mm Tris, $150 \mathrm{~mm} \mathrm{NaCl}$, and 0.1\% Tween 20), incubated with anti-P60 or anti-P80 antibody diluted 1:20,000 in TTBS with $5 \%$ milk, washed, and reacted with goat anti-rabbit IgG conjugated with HRP (Jackson ImmunoResearch, West Grove, PA). To assure the accuracy of readings, each gel was also loaded with 20-160 fmol of $\mathrm{P} 60$ or $20-80 \mathrm{fmol}$ of $\mathrm{P} 80$ standard protein. After reaction with chemiluminescent peroxidase substrate (Super Signal; Pierce), each blot was covered with x-ray film. For each blot, we used at least three exposure times $(5,15$, and $30 \mathrm{~s})$ to obtain the best bands for subsequent imaging and OD readings. P60 or P80 concentrations were read from standard curves drawn for each blot on which all sample proteins and standard proteins were visualized under identical conditions.

Films were imaged using an Epson Perfection 1240U scanner. The optical densities were measured for bands corresponding to full-length P80 and P60 katanin using a Macintosh (Apple Computer, Cupertino, CA) computer running the public domain NIH Image (http://rsb.info. nih.gov/nih-image/). Protein concentrations were read from standard curves. P60/P80 ratios were calculated by dividing P80 concentration (in femtomoles) by $\mathrm{P} 60$ concentration (in femtomoles).

In situ hybridization of brain sections. Sense and antisense riboprobes for P80 katanin were synthesized with a digoxigenin (DIG) RNA labeling kit (Boehringer Mannheim, Mannheim, Germany) precisely as we described for P60 katanin (Karabay et al., 2004). A template for the production of nonradioactive riboprobes for in situ hybridization was made by subcloning the full-length P80 katanin into the pBluescript SK-plasmid (Stratagene). After template linearization, sense and antisense digoxigenin-labeled riboprobes were transcribed with a ribonucleotide mix containing digoxigenin-UTP (Boehringer Mannheim) and either T3 or T7 RNA polymerase (Promega, Madison, WI) according to the instructions of the manufacturer. Frozen sections $(16-20 \mu \mathrm{m})$ were collected and in situ hybridized for $21 \mathrm{~h}$ at $55^{\circ} \mathrm{C}$ in a humid chamber using $200 \mathrm{ng} / \mathrm{ml}$ DIG-labeled riboprobes in hybridization buffer containing $50 \%$ formamide and $10 \%$ dextran sulfate. Sections were then washed in $2 \times$ SSC at $20^{\circ} \mathrm{C}$ for $1 \mathrm{~h}$ and in $0.1 \times$ SSC at $65^{\circ} \mathrm{C}$ for $1 \mathrm{~h}$. Hybrids were detected using anti-DIG antibody conjugated with alkaline phosphatase (Boehringer Mannheim). Labeled sections were imaged using a Zeiss (Oberkochen, Germany) 135TV inverted microscope equipped with a high-resolution CCD camera and a Toshiba computer running MetaMorph software (Universal Imaging Corporation, Downingtown, PA).

Immunostaining of tissue sections. Mouse brains at various stages of development were sectioned on a cryostat and prepared for single-label visualization of P60-katanin or P80-katanin immunoreactivity using a standard peroxidase technique (Solowska et al., 2002; Karabay et al., 2004). Brains from at least three mice at each stage were serially sectioned and immunolabeled. Sections were treated with $0.2 \% \mathrm{H}_{2} \mathrm{O}_{2}$ in TBST $(100$ mм Tris-HCl, pH 7.5, 150 mм NaCl, 0.1\% Tween 20), blocked with 5\% skim milk in TBS, and incubated overnight with anti-P60-katanin or anti-P80-katanin antibody and then exposed to anti-rabbit secondary antibody conjugated with HRP (Jackson ImmunoResearch). HRP activity was detected using $0.5 \mathrm{mg} / \mathrm{ml}$ diaminobenzidine, $0.006 \% \mathrm{H}_{2} \mathrm{O}_{2}$, and $0.1 \%$ nickel ammonium sulfate in $50 \mathrm{~mm}$ Tris, $\mathrm{pH}$ 7.5. Sections were imaged as described above (see In situ hybridization of brain sections).

Preparation, transfection, and immunolabeling of cell cultures. Cultures 
of rat hippocampal neurons and RFL6 rat fibroblasts were prepared as described previously (Yu and Baas, 1994; Buster et al., 2003). For quantification of P60- and P80-katanin levels in various developmental stages of the cultured hippocampal neuron, the cultures were fixed at 1, 2, 3, 4, $5,6,7,10$, or 12 d. For transfection of the katanin constructs, we used either a modified electroporation device called a Nucleofector (Amaxa Biosystems, Cologne, Germany) before plating or Lipofectamine 2000 (11668-027; Invitrogen) at $24 \mathrm{~h}, 48 \mathrm{~h}, 72 \mathrm{~h}$, or $6 \mathrm{~d}$ after plating the cells. For the former, the single-cell suspension of hippocampal neurons or RFL6 cells was resuspended in Nucleofector solution (Amaxa Biosystems), and the cells were then transfected with EGFP alone (control) or with one of the various katanin constructs using the nucleofection device according to instructions of the manufacturer and recommended settings (Karabay et al., 2004). After nucleofection, the neurons were plated onto poly-L-lysine-treated glass coverslips as described previously ( $\mathrm{Yu}$ and Baas, 1994), whereas the RFL6 cells were transferred to $37^{\circ} \mathrm{C}$ RFL6 culture medium (F12K plus 20\% FBS) and plated on untreated glass coverslips. For transfections on neurons in the culture dish at various stages of their development, we used Lipofectamine 2000. For this procedure, 2-4 $\mu \mathrm{g}$ of DNA and 5-10 $\mu \mathrm{g}$ of Lipofectamine 2000 were used per $35 \mathrm{~mm}$ dish. The ratio of DNA to Lipofectamine 2000 was 1:2.5. The cells were incubated in the DNA/Lipofectamine medium for $5 \mathrm{~h}$. After transfection, neurons were transferred to $37^{\circ} \mathrm{C}$ hippocampal neuron plating medium (Neurobasal medium supplemented with 2\% B27, 0.3\% glucose, $1 \mathrm{~mm}$ glutamine, and 5\% FBS). Transfection efficiency was generally $5-10 \%$ in neurons and $15-30 \%$ in RFL6 cells, with EGFP fluorescence appearing within the first several hours after transfection. The plasmids used were EGFP (control), pEGFP-C1-P60, pEGFP-C1-P80, and pEGFP-C1-con80.

For immunofluorescence studies on microtubule distribution, cultures were briefly washed with $\mathrm{PBS}$ at $37^{\circ} \mathrm{C}$ and then simultaneously fixed and extracted with $0.2 \%$ glutaraldehyde and $0.1 \%$ Triton X-100 for 15 min. Cultures were washed with PBS/0.1\% Tween 20 for 5 min three times, quenched with $10 \mathrm{mg} / \mathrm{ml}$ sodium borohydride for $10 \mathrm{~min}$ three times, and then blocked with $10 \%$ normal goat serum and $10 \mathrm{mg} / \mathrm{ml} \mathrm{BSA}$ in PBS/0.1\% Tween 20 for $1 \mathrm{~h}$. After blocking, cultures were exposed to an anti-EGFP rabbit polyclonal antibody (1:500; ab6556-25; Abcam, Cambridge, MA) overnight at $4^{\circ} \mathrm{C}$. (Enhancement of EGFP fluorescence by immunofluorescence assisted in discerning the cells expressing EGFP, EGFP-P60-katanin, EGFP-P80-katanin, and EGFP-con80-katanin constructs from those that were not expressing, given that the fluorescence of the EGFP itself tended to diminish during the fixation and extraction.) Cultures were then rinsed three times in PBS for $5 \mathrm{~min}$, followed by exposure to an anti- $\beta$-tubulin antibody directly conjugated to cyanine 3 (Cy3) (1:300; C4585; Sigma) together with Alexa Fluor 488-conjugated secondary goat anti-rabbit antibody (1:200; A11008; Molecular Probes, Eugene, OR). Cells were then rinsed extensively in PBS and mounted in a medium that reduces photobleaching. Images were obtained using a Pascal confocal microscope (Zeiss).

For immunofluorescence studies on P60-katanin and P80-katanin levels in RFL6 cells and hippocampal neurons, cells were fixed with $0.2 \%$ glutaraldehyde and then postextracted with $0.1 \%$ Triton X-100, and cultures were double labeled by exposure first to our P60-katanin polyclonal antibody (1:1000) or P80-katanin polyclonal antibody (1:500) overnight at $4^{\circ} \mathrm{C}$ and then to Alexa Fluor 488-conjugated goat anti-rabbit (A11008; Molecular Probes) and Cy3-conjugated $\beta$-tubulin mouse monoclonal antibody for $1 \mathrm{~h}$ (at 1:200 and 1:400, respectively). Images were obtained on an Axiovert 200 microscope (Zeiss) equipped with a high-resolution CCD (Orca ER; Hamamatsu, Shizouka, Japan) or a Pascal confocal microscope (Zeiss). All images were obtained using identical camera, microscope, and imaging criteria such as gain, brightness, contrast, and exposure time. Efforts were made to ensure that the images were not saturated and that minimal bleaching of the fluorescence occurred before image acquisition. Digital gray values of image pixels representing arbitrary fluorescence units (AFUs) were obtained using the AxioVision software. Fluorescence intensity was quantified for cells that had not been induced to express any of the katanin constructs, cells that had been induced to express EGFP alone, and cells that had been induced to express the various katanin constructs. For hippocampal neurons, quanti- fication was performed on the cell body, immature ("minor") processes, axon, and dendrite. For interphase RFL6 cells, quantification was performed on the entire cell. For the developmental analyses on cultured neurons, we quantified the ratio of fluorescence intensity obtained with the P60-katanin or P80-katanin antibody to the fluorescence intensity obtained with the antibody to $\beta$-tubulin. For both the developmental studies and the studies involving overexpression of the katanin constructs neuronal, we quantified the entire cell body. For the processes (axons, dendrites, and immature processes) and their tips, we quantified a similar sized region in all cases. In each case, the average AFU within the cell or the compartment of the neuron was obtained. All experiments were replicated four times, with 30 neurons quantified each time. Statistics were done using the Student's $t$ test.

\section{Results}

The nucleotide sequence for rat P80 katanin was obtained and submitted to GenBank (accession number AY953248). There is $88 \%$ identity with the human nucleotide sequence, $94 \%$ identity with the mouse sequence, and $82 \%$ identity with the Xenopus sequence for P80 katanin. Two polyclonal antibodies generated against fusion proteins derived from the rat cDNA were monospecific on Western blots and labeled the mitotic spindle in both human and rat fibroblasts (data not shown), as was the case with the P60-katanin antibody that we generated previously (Karabay et al., 2004). One of the P80 antibodies was better suited for Western blot analysis, whereas the other gave a stronger signal on samples fixed for immunostaining.

\section{Quantitative studies on the levels and ratio of P60 and P80 katanin in various tissues}

It is generally assumed that P60 katanin forms a heterodimer with P80 katanin. On this basis, it would be reasonable to believe that the P60 and P80 subunits are present within cells at equal levels, although this would not have to be the case. To investigate this matter, we quantitatively analyzed the levels of the two subunits in various tissues of the developing rat using Western blots. To assure accurate measurements, we collected and combined E15 and E18 tissues from 12 individual animals at each stage, P0 and P6 tissues from six to eight animals, and P12 and adult tissues from four to six animals. Therefore, the P60 and P80 concentrations calculated for different tissues at different developmental time points represent population averages rather than individual animals. To determine P60/P80 ratios, we performed 20 quantitative Western blots (10 for P60 and 10 for P80 simultaneously). Tissue collected at each time point was tested three to four times (see Materials and Methods).

As expected, the levels of P60 and P80 katanin vary developmentally in both neuronal and non-neuronal tissues. Interestingly, we found that the levels of P60 and P80 katanin are far from equimolar (Fig. 1). Expression of both proteins is higher in embryos and newborns than at later postnatal stages. This finding is consistent with the importance of katanin in rapidly proliferating embryonic tissues and in early postmigratory neurons from which axons begin to grow. In non-neuronal tissues [lungs (data not shown) and heart] (Fig. $1 \mathrm{~B}$ ), the levels of P60 katanin are always much higher (10-20 times) than the levels of P80 katanin. In the CNS [cerebral cortex (Fig. 1C), hippocampus (Fig. 1D), cerebellum, and spinal cord (data not shown)], the P60/P80 ratios are lower (0.7-7 times). This is because P80 expression in the CNS is two to four times higher than in non-neuronal tissues, and because P60 levels in later postnatal stages are lower. Higher levels of P60 in postnatal, non-neuronal tissues might reflect the presence of proliferating epithelial cells. Interestingly, in the CNS, relatively higher levels of P60 were detected in adult hippocam- 
A

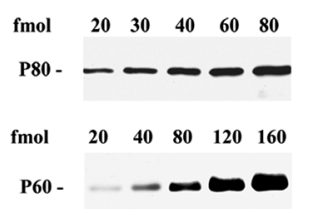

B

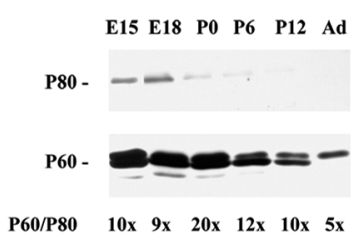

C

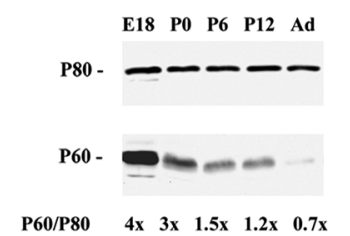

D

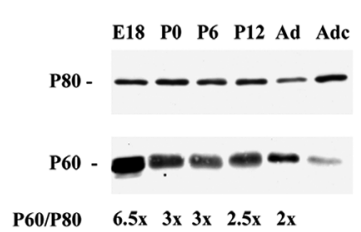

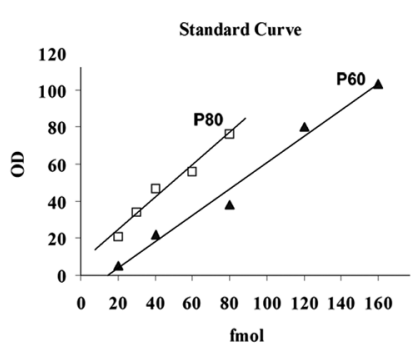

HEART

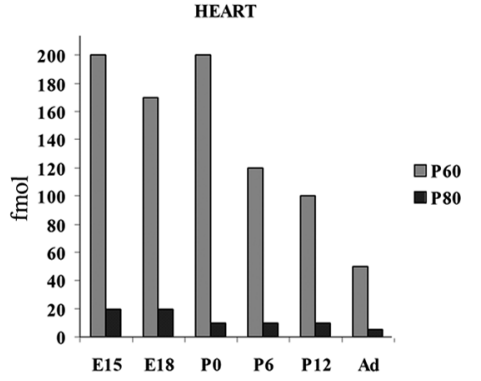

CEREBRAL CORTEX
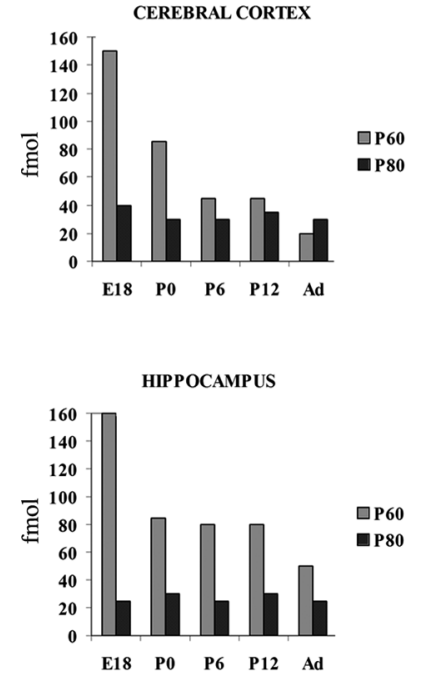

Figure 1. Biochemical determination of the ratio of P60 to P80 katanin. $A$, Calibration of anti-P60 and anti-P80 antibodies. Standard curves were obtained using $20-160 \mathrm{fmol}$ of $\mathrm{P} 60$ or $20-80 \mathrm{fmol}$ of P80 katanin. $\boldsymbol{B}-\boldsymbol{D}$, Concentration of P80 and P60 katanin in developing rat tissues. Tissues were collected and combined from 12 E15 and 12 E18 rats, six to eight PO or P6 rats, and four to six P12 and adult (Ad) rats. Protein extracts from $1000 \mu \mathrm{g}$ of combined tissues from different developmental stages were prepared in sample buffer. One-half of the extract was used for Western blot analysis with anti-P80 antibody, and the other half was used simultaneously for Western blot analysis with anti-P60 antibody. The optical densities were measured for bands corresponding to full-length P80 and P60 katanin, and protein concentrations were read from standard curves. Each bar in $\boldsymbol{B}-\boldsymbol{D}$ represents the average amount of katanin found in tissue from 4-12 animals. P60/P80 ratios were calculated by dividing P80 concentration (in fmol) by P60 concentration (in fmol). $\boldsymbol{B}$, Concentration of P80 and P60 katanin in developing rat heart. $C$, Concentration of $\mathrm{P} 80$ and $\mathrm{P} 60$ katanin in developing rat cerebral cortex. D. Concentration of P80 and P60 katanin in developing rat hippocampus. Even when total protein concentration in the adult cerebral cortex sample (Adc) was two times higher than in the adult hippocampus sample (Ad), the P60 concentration was still clearly higher in the hippocampus.

pus (which is known for neuronal plasticity) compared with adult cortex (Fig. 1D). However, differences in concentration of P60 in hippocampus and cerebral cortex might also result from a dilution effect caused by a larger amount of white matter in the cerebral cortex.

Immunostaining and in situ hybridization on neuronal and non-neuronal tissue sections revealed additional details of P60 and P80 expression. The levels of P60 katanin in the embryo are slightly higher in non-neuronal tissues than in neuronal tissues ( $\sim 200 \mathrm{fmol} / 500 \mu \mathrm{g}$ of embryonic heart or lungs compared with $\sim 160 \mathrm{fmol} / 500 \mu \mathrm{g}$ of embryonic CNS tissue). However, P60 and P80 are distributed uniformly among the cells that comprise the developing heart (Fig. 2G,H) and lungs (data not shown) but are unevenly expressed in different subsets of cells in the developing CNS (Figs. 2E, $F, 3 A-H$ ). It is noteworthy in this regard that the concentration of P60 is similar per $500 \mu \mathrm{g}$ of neuronal and nonneuronal tissue, but that the concentration of P80 is higher in CNS compared with other tissues. Therefore, the concentration of both proteins in subsets of expressing CNS cells is significantly higher than that in non-neuronal tissues [compare the intensity and distribution of the immunostaining for P60 and P80 in the cerebral cortex (Figs. 2E,, $3 A-D$ ) with that in the heart (Fig. $2 G, H)]$. In mouse E13 cerebral cortex, the expression of both P60 and P80 is intense near ventricles where radial glia and recently born neurons are located (Fig. 2 E, F). By E16, the highest levels of P60 and P80 are observed in postmigratory neurons of early cortical plate (Fig. $3 A-D$ ). In situ hybridization also shows that in the developing hippocampus, both $\mathrm{P} 60$ and P80 are present in neurons located in the dentate gyrus and Ammon's horn (Fig. 3G, $H$ ). Immunostaining also revealed the presence of P60 and P80 katanin in PNS axons. Figure 2, $A$ and $B$, shows katanin in axons exiting the fifth cranial nerve, and $C$ and $D$ show katanin in dorsal root ganglion (DRG) axons. As in the CNS, P60 expression in PNS axons is higher than that of P80. Unlike the case with P60, there is no marked increase in P80 levels during phases of rapid axonal growth.

\section{Distribution and levels of P60 and P80 katanin in cultured hippocampal neurons}

Cultured rat hippocampal neurons have proven to be extremely useful for studies on neuronal development, because they progress through a series of stereotyped stages of differentiation in the culture dish. As defined by Dotti et al. (1988), stage 1 is the initial formation of lamellas, stage 2 is the coalescence of the lamellas into multiple immature ("minor") processes, stage 3 is when one of the immature processes becomes an axon, stage 4 is when the other immature processes become dendrites, and stage 5 is when the axons and dendrites mature and develop synapses. The neurons are typically in stage 3 by the end of the next day after culturing and typically in early stage 4 by the fourth day. Unlike with the quantitative Western blot analysis, we could not use immunofluorescence to directly compare the absolute levels of P60 and P80 katanin in these cultures. Therefore, we double labeled the cultures at various stages of development with an antibody to one of the two katanin subunits and $\beta$-tubulin and used quantitative immunofluorescence to obtain a ratio of the katanin subunit to the $\beta$-tubulin within the cell body, within the processes, and at the tips (growth cones) of the processes. Figure 4 shows examples of such cultures with katanin in green and $\beta$-tubulin in red. Figure $4 A-D$ show P60, whereas Figure $4 E-H$ show P80. Figure 5 shows higher-magnification images of neurons in early stage 4 with P60 shown in Figure 5, $A$ and $B$, and P80 shown in $C$ and $D$. The quantification is shown graphically in Figure 5E-J. In general, P80 is far more enriched in cell bodies, where its ratio to tubulin is approximately six times the ratio in the processes. This might relate, in part, to the fact that $\mathrm{P} 80$ has a centrosomal targeting function (McNally et al., 2000). P60, in 


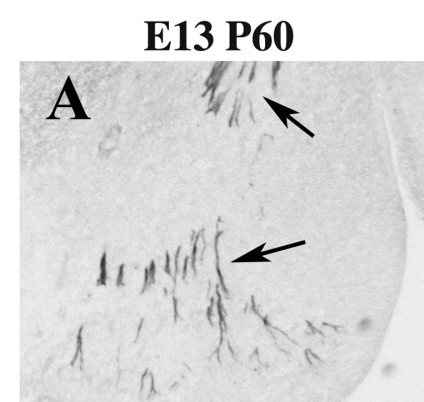

5TH CRANIAL N.
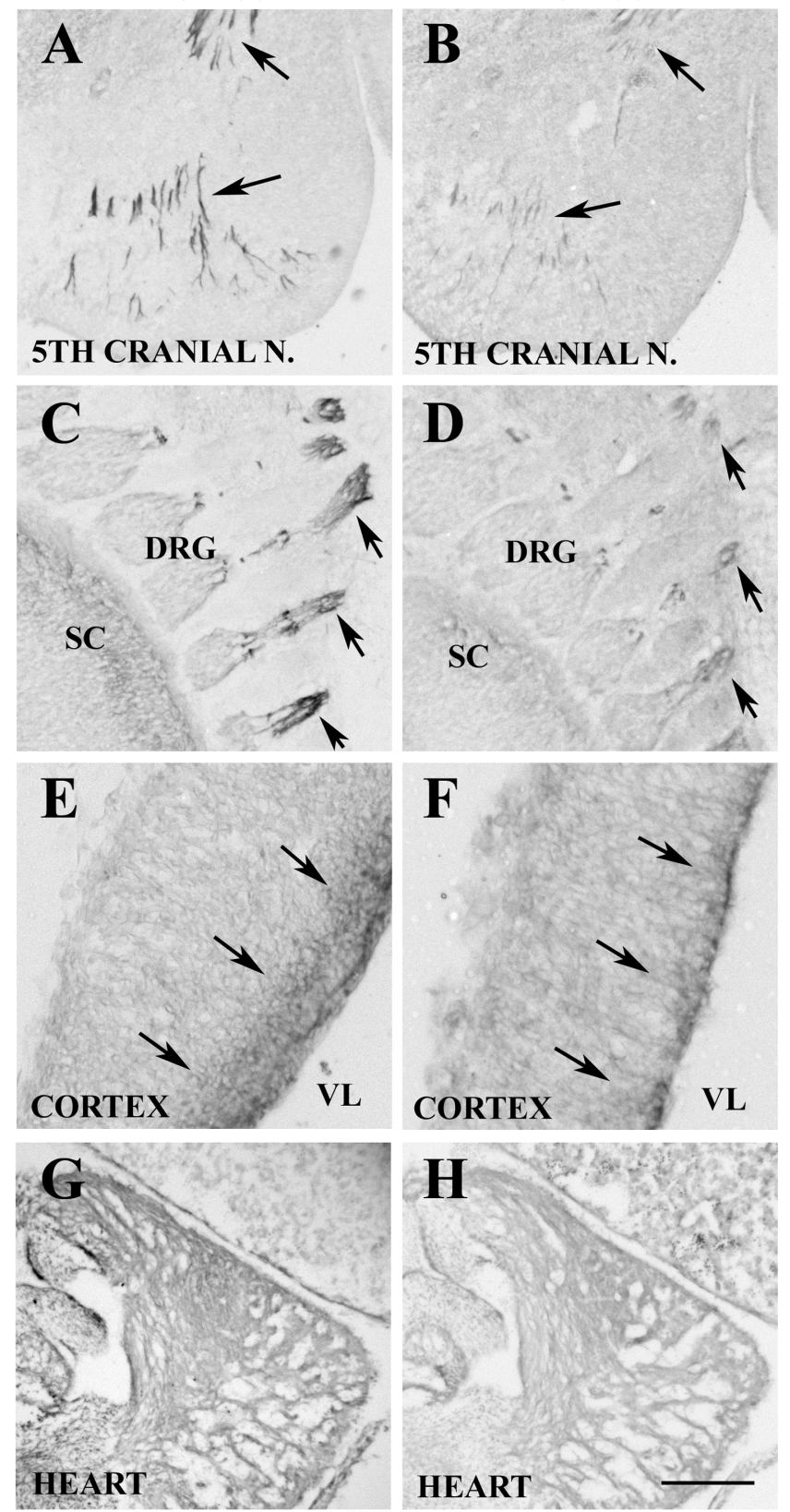

Figure 2. Expression of $\mathrm{P} 60$ and $\mathrm{P} 80$ katanin in neuronal and non-neuronal tissues. $\boldsymbol{A}, \boldsymbol{C}, \boldsymbol{E}, \boldsymbol{G}$, E13 mouse sections immunolabeled for $\mathrm{P} 60$ katanin. $\boldsymbol{B}, \boldsymbol{D}, \boldsymbol{F}, \boldsymbol{H}, \mathrm{E} 13$ mouse sections immunolabeled for P80 katanin. $\boldsymbol{A}$, High levels of P60 katanin are present along the lengths of axons exiting the fifth cranial nerve (arrows). $\boldsymbol{B}$, Expression of P80 katanin in axons exiting the fifth cranial nerve is considerably lower than that of P60 katanin (arrows). C, P60 katanin expression is abundant in peripheral processes (arrows) of DRG sensory neurons. $D$, The same processes of DRG sensory neurons express P80 katanin (arrows) but at a significantly lower level. E, AtE13 in the developing cerebral cortex, high levels of P60 katanin are present only in cells and processes near the lateral ventricle (arrows). Labeled cells are radial glia or recently born neurons. F, P80 katanin is also present mostly in cells near the ventricle in E13 cortex (arrows). $\mathbf{G}, \boldsymbol{H}, \mathrm{In} E 13$ heart, both P60 and P80 katanin are more uniformly distributed in developing cardiac muscle cells. Scale bar: $\boldsymbol{A}-\boldsymbol{D}, \boldsymbol{G}-\boldsymbol{H}, 230 \mu \mathrm{m} ; \boldsymbol{E}, \boldsymbol{F}, 115 \mu \mathrm{m}$. 5TH CRANIAL N., Fifth cranial nerve; VL, lateral ventricle; $S C$, spinal cord.

contrast, is not so markedly higher in cell bodies compared with processes, where in most cases the ratio is less than double the ratio in the processes. P80 is also more evenly distributed within the processes than P60 and thus does not stand out as well against

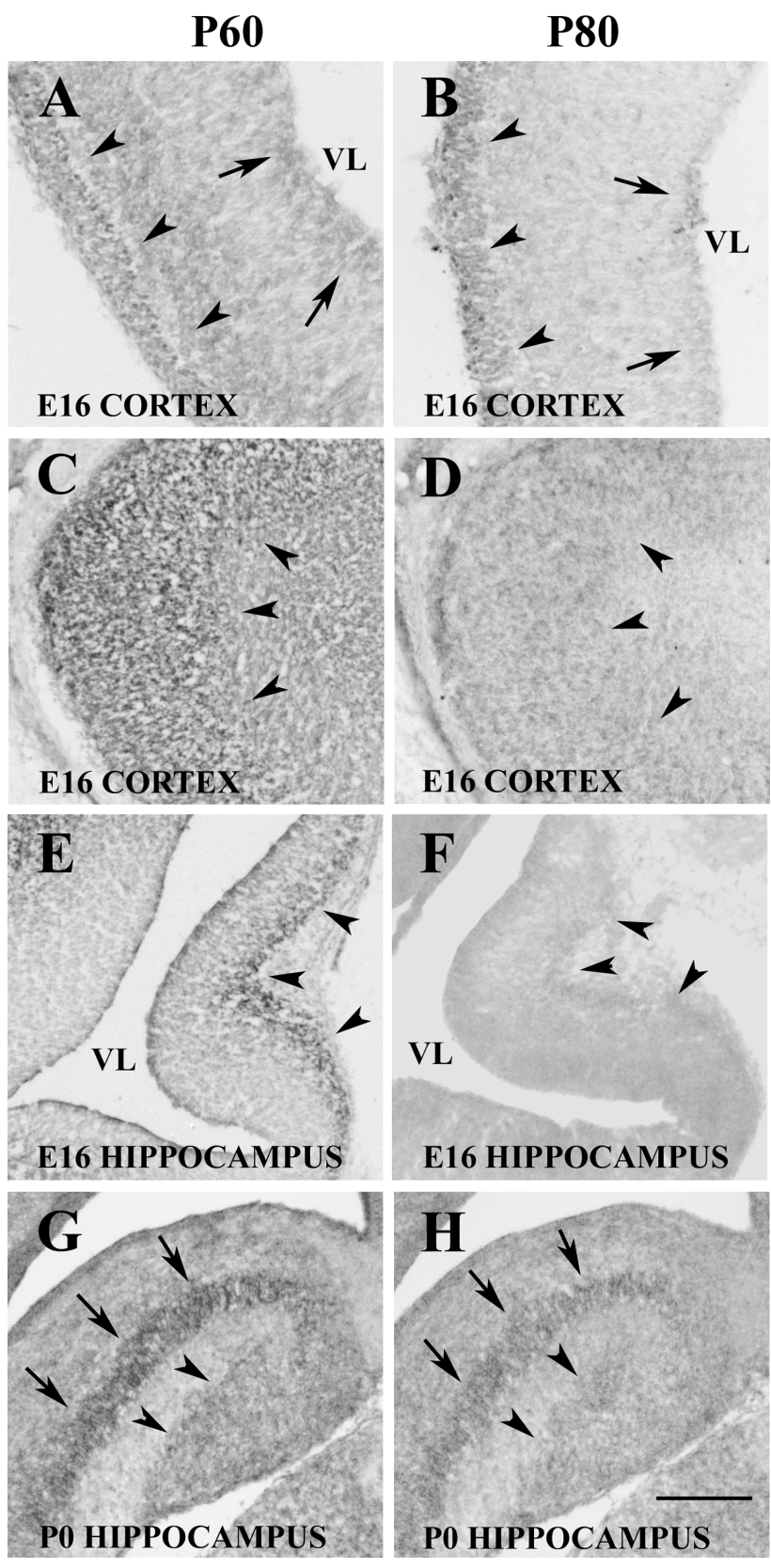

Figure 3. Expression of $\mathrm{P} 60$ and $\mathrm{P} 80$ katanin in the cerebral cortex and hippocampus. $\boldsymbol{A}, \boldsymbol{C}, \boldsymbol{E}$, E16 mouse cerebral cortex sections $(\boldsymbol{A}, \boldsymbol{C})$ and an E16 mouse hippocampus section $(\boldsymbol{E})$ immunolabeled for P60 katanin. $\boldsymbol{B}, \boldsymbol{D}, \boldsymbol{F}$, E16 mouse cerebral cortex sections $(\boldsymbol{B}, \boldsymbol{D})$ and an E16 hippocampus section $(\boldsymbol{F})$ immunolabeled for P80 katanin. $\mathbf{G}$, PO hippocampus section in situ hybridized with a riboprobe specific for $\mathrm{P} 60$ katanin. $\boldsymbol{H}, \mathrm{P} 0$ hippocampus section in situ hybridized with a riboprobe specific for P80 katanin. $A$, By E16, P60 is detectably expressed in cells adjacent to the ventricle (arrows) but also in cells far from ventricular zones, which are postmigratory neurons of the early cortical plate (arrowheads). B, P80 expression is very weak in cells adjacent to ventricle (arrows) but is clearly detectible in cells that have migrated toward the pia (arrowheads). C, D, The P60 and P80 expression, respectively, is elevated in these sections within superficial layers of the E16 cerebral cortex, where the postmigratory neurons are localized. $\boldsymbol{E}, \boldsymbol{F}$, P60 katanin expression and P80 expression in E18 hippocampus is also high in early postmigratory neurons (arrowheads). G, $\boldsymbol{H}$, At postnatal day 1 (P0), both P60 and P80 are highly expressed in layers containing hippocampal neurons, including the dentate gyrus (arrowheads) and Ammon's horn (arrows). Scale bar: $\boldsymbol{A}-\boldsymbol{D}, \mathbf{G}, \boldsymbol{H}, 230 \mu \mathrm{m} ; \boldsymbol{E}, \boldsymbol{F}, 115 \mu \mathrm{m}$. VL, Lateral ventricle.

the red coloration of the microtubules in the pseudocolored overlays. The levels of P80 do not markedly vary in the different types of processes, except for in the late dendrites, in which the levels are approximately triple those in the early immature pro- 

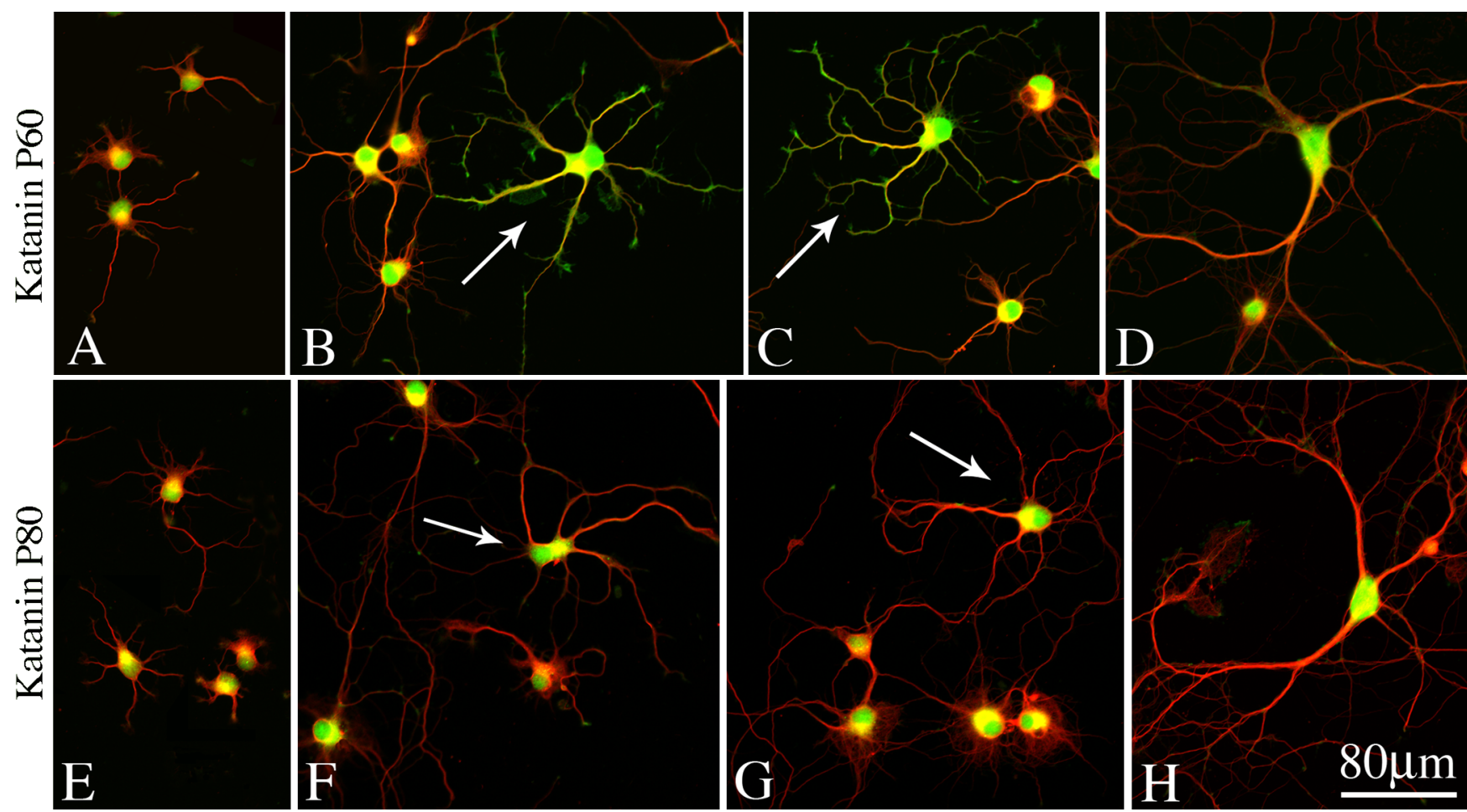

Figure 4. Comparison of $\mathrm{P} 60$ and $\mathrm{P} 80$ katanin expression patterns in developing cultured hippocampal neurons. Microtubule staining ( $\beta$-tubulin) is shown in red, and katanin staining is shown in green. The top row shows $\mathrm{P} 60$ katanin $(\boldsymbol{A}-\boldsymbol{D})$, and the bottom row shows $\mathrm{P} 80$ katanin $(\boldsymbol{E}-\boldsymbol{H})$. P60 and P80 are both present throughout all compartments of the neuron; processes that appear red-orange have lower levels of katanin, whereas processes that appear green have higher levels of katanin. $\boldsymbol{A}, \boldsymbol{E}$, Neurons in stage $2 / 3$ before dendritogenesis. $\boldsymbol{B}, \boldsymbol{C}, \boldsymbol{F}, \boldsymbol{G}$, Neurons in early stage 4 (arrows). $\boldsymbol{D}, \boldsymbol{H}$, Neurons in late stage 4 . Note that P80 is more concentrated in cell bodies compared with P60. Note that P60 levels are dramatically higher during early stage 4 compared with earlier and later developmental stages. No such spike in expression is observed with P80. Scale bar, $80 \mu \mathrm{m}$.

cesses. With regard to P60, there are spikes in the levels in the cell body, the axon, and the dendrites at early stage 4, with the levels coming back down in late stage 4. At all developmental stages, there is slightly more P80 at the tips of the processes compared with the processes themselves. With regard to P60, there is no significant difference between the tips and the shafts of the immature processes and the stage 3 axons but very dramatic enrichment at the tips of both axons and dendrites in early stage 4, wherein the ratio jumps to severalfold higher that even in the cell body (see Fig. 5 for details and statistics).

\section{Effects of P60- and P80-katanin constructs}

Before manipulating P60 and P80 katanin in hippocampal neurons, we directly compared the effects of the katanin constructs on microtubule severing in RFL6 rat fibroblasts, in which microtubule lengths can be discerned easily. Previous studies suggest that ectopically expressed katanin proteins are functionally active, presumably to a similar degree as their endogenous counterparts (McNally et al., 2000, 2002; Karabay et al., 2004). We also expressed our rat construct for the truncated version of P80, called con80, which contains the region thought to augment the severing properties of P60 (McNally et al., 2000). As shown in Figure 6, an increase in P60 levels of $\sim 30 \%$ caused an $\sim 60 \%$ reduction in the microtubule mass, whereas an increase in P80 levels (or con 80 ) of $\sim 500 \%$ caused only an $\sim 16 \%$ reduction in microtubule mass. (Note that there is no endogenous con 80 , so the elevation is relative to the levels of endogenous full-length P80). However, despite the fairly modest diminution in microtubule mass, the distribution of microtubules is markedly altered as a result of the expression of the $\mathrm{P} 80$ and con 80 constructs; there is no longer a focused concentration of microtubules in the centro- somal region. These results are consistent with an enhancement of the severing of microtubules from the centrosome with the P80 and con80 constructs; however, unlike with the P60 construct, there is still an abundance of long microtubules. These results suggest that elevation of P 80 augments microtubule severing but, even at much higher levels, does not produce nearly as dramatic an effect on the severing as the P60 construct and does not cause such a substantial loss of polymer mass. The fact that we obtained essentially the same result with the P80 and con 80 constructs indicates that this effect is not caused by an enhanced targeting of katanin to the centrosome, given that con 80 lacks the centrosomal targeting domain.

Next, we sought to determine the effects of overexpressing the katanin constructs in hippocampal neurons. As with the fibroblasts, we selected cells that were highly expressing the constructs. There was no indication of cell death, although it is conceivable that the most highly expressing neurons died before we observed the cultures. Unlike our previous studies in which expression was only performed at the time of plating, we found that we could successfully use transfection reagents to express the constructs at various developmental stages in these neurons. The results were particularly interesting when we overexpressed the constructs for only $1 \mathrm{~d}$ at various periods of time after plating. Figure $7 A-D$ shows $1 \mathrm{~d}$ of katanin P80, con80, and P60 overexpression after the neuron had been plated for $1 \mathrm{~d}$ (thus referred to as a "day 2" neuron). The control neuron, expressing EGFP alone, is in stage 3 , with one long axon and multiple short immature processes. Comparable neurons expressing either P80 or con 80 have many more immature processes together with a single axon. In contrast, the neuron overexpressing $\mathrm{P} 60$ has far fewer processes, and it is unclear whether or not one of them is a bona fide axon. 

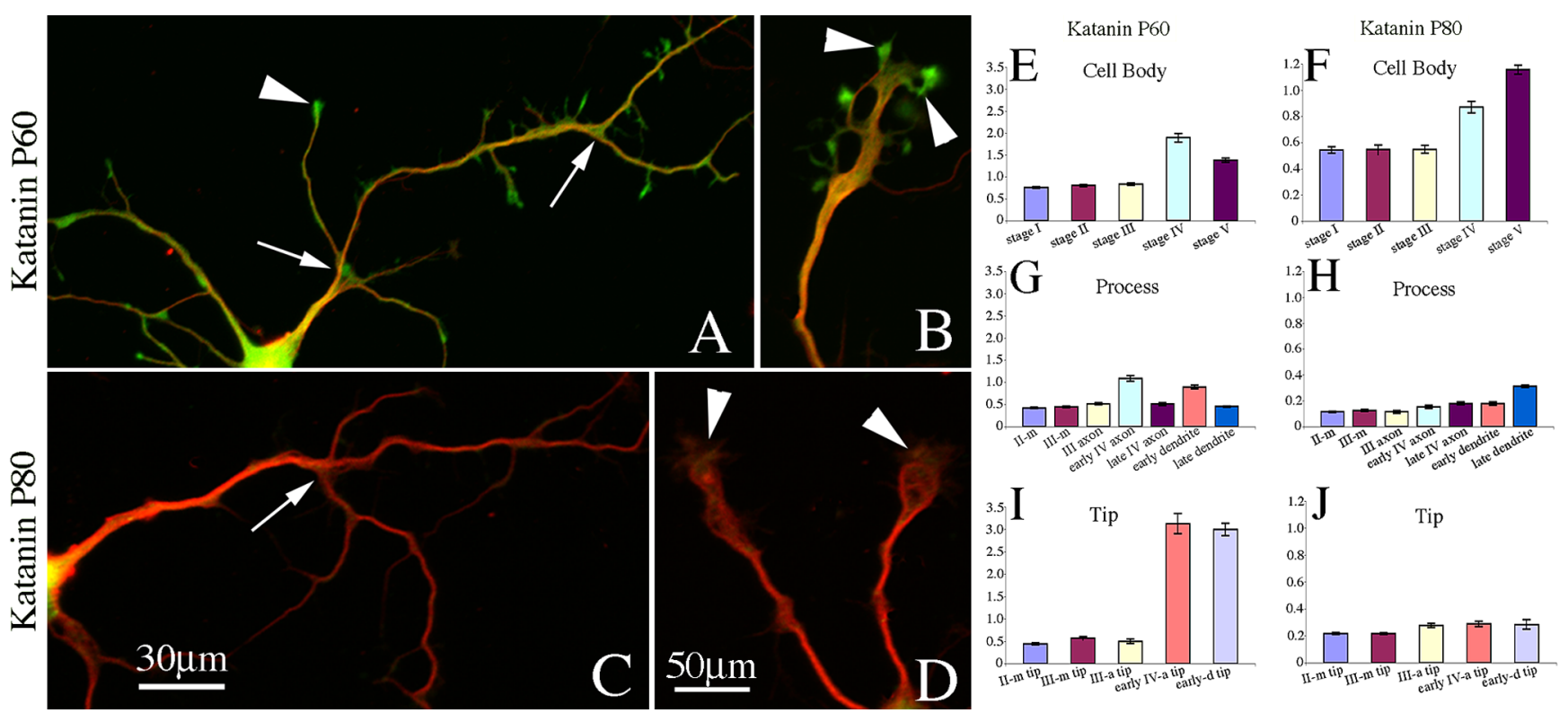

Figure 5. Quantification of endogenous $P 60$ and $P 80$ katanin levels in cell bodies, processes, and tips of processes of cultured hippocampal neurons at different developmental stages. $A-D$, Neurons in early stage 4. Microtubule staining ( $\beta$-tubulin) is shown in red, and katanin staining is shown in green. $\boldsymbol{A}, \boldsymbol{B}, \mathrm{P} 60$ katanin. $C, \boldsymbol{D}, \mathrm{P} 80 \mathrm{katanin}$. Arrowheads point to growth cones, which are notably enriched for P60 but not P80. $A$ and $\boldsymbol{C}$ are lower magnification and $\boldsymbol{B}$ and $\boldsymbol{D}$ are higher magnification of the tip regions (growth cone) of axons. The arrows point to branch points, which were also somewhat enriched for P60 but not P80. Quantification of data obtained from various types of processes and various regions of the neuron are shown in $E$-J. Data ( $y$-axis) are expressed as the ratio of P60 or P80 obtained with each antibody to $\beta$-tubulin calculated using AFUs. Absolute levels of P60 and P80 cannot be compared with this approach. Note that the two subunits of katanin do not show identical changes in the various compartments (see Fig. 4). Stages of development [as defined by Dotti et al. (1988)] are shown as roman numerals in these graphs. There is a gradual increase in P80 levels within the cell body as the neuron transitions into early and late stage $4(1.6-2.1$ times compared with stage $1 ; p<0.001)$. Compared with stage 3 axons, the levels of P80 in early and late stage 4 axons only increased by $1.36-1.5$ times $(p<0.05)$. In late dendrites, the levels are approximately triple those in the early immature processes $(p<0.001)$. With regard to $\mathrm{P} 60$, there is a sharp increase in early stage 4 cell bodies, axons $(\sim 2.5$ times; $p<0.001)$, and dendrites $(\sim 2.25$ times; $p<0.001)$ compared with stage 1 . The levels of P60 came down in the late stage 4 cell bodies ( 1.8 times; $p<0.001)$, axons ( 1.25 times; $p<0.05)$, and dendrites $(p>0.05)$ compared with stage 1 . At all developmental stages, there is more $P 80$ at the tips of the processes compared with the processes themselves $(1.6-2.5$ times; $p<0.01)$. With regard to $\mathrm{P} 60$, there is no significant difference between the tips and the shafts in the immature processes and the stage 3 axons ( $p>0.05$ ), but there is a very dramatic enrichment at the tips of both axons and dendrites in early stage 4 ( $3-6$ times; $p<0.0001)$. Scale bar: $A, C, 30 \mu \mathrm{m} ; \boldsymbol{B}, \boldsymbol{D}, 50 \mu \mathrm{m}$.

Quantification of process length and number shows that particular developmental stages are more sensitive to katanin manipulation (Fig. 7G,H). The second day in culture is noteworthy, because there is a marked diminution in process number with P60 overexpression but a marked increase in process number with overexpression of $\mathrm{P} 80$ or con80. The increase in total process number with the P80 constructs together with an overall decrease in total process length of $30 \%$ reflects the fact that the axon achieved a shorter average length compared with controls. The fourth day in culture (corresponding to early dendritogenesis; early stage 4) is particularly sensitive in terms of a diminution in total process length and process number; this is the case when any of the katanin constructs are expressed (Fig. 7G,H) (see figures for details and statistical analyses of the data).

Regional differences in the susceptibility of microtubules to the katanin constructs

Quantification of microtubule levels in different regions of the neurons at different developmental stages (again, with the katanin constructs expressed for only $1 \mathrm{~d}$ ) revealed marked differences in the susceptibility/resistance of the microtubules in different compartments of the neuron-to-katanin-induced loss. Figure $8, A a$ and $B b$, shows late stage 3 neurons (third day in culture) with a single axon together with multiple immature processes that have not yet begun to show indications of dendritic differentiation. Neurons were immunostained to reveal microtubules and are shown in the figure as the original fluorescence image together with a pseudocolored image to accentuate differences in fluorescence intensity. Control neurons expressing
EGFP alone displayed robust microtubule staining in the cell body, axon, and immature processes (Fig. $8 A a$ ). Neurons expressing the P60-katanin construct showed marked diminution in microtubule levels within the cell body and minor processes but no such diminution in the axon (Fig. $8 \mathrm{Bb}$ ). Other axons showed somewhat more loss but never to the degree observed in the cell body and immature processes. It was not uncommon to observe twisty bundles of compressed microtubules in the neurons expressing any of the katanin constructs, although the significance of this observation is unknown at present (Fig. $8 \mathrm{Bb}$, small arrows). Quantitative results of stage 3 neurons are shown graphically in Figure $8 C c$. Interestingly, the P80 constructs produced nearly as much loss of microtubule mass as the P60 construct in the cell body and immature processes but caused no detectable loss whatsoever of microtubules from the axon. In the axon, P60 overexpression caused an average 20\% loss of microtubules, which is less than the degree that it caused in cell bodies $(58 \%)$ or minor processes $(37 \%)$. These results indicate that the microtubules within early axons are less sensitive to katanininduced severing/depolymerization than microtubules in other compartments of the neuron.

At stage 4, shown in Figure $8 D d$, the various katanin constructs caused a loss of microtubules from the cell body compared with their effects at stage 3 . In the stage 4 axons, like stage 3 axons, P80 or con 80 did not cause any microtubule diminution. However, at stage 4 , there was also no diminution in microtubule levels in the axon by P60, suggesting that microtubules in more developed axons have even greater resistance to katanin-induced severing/depolymerization than microtubules in early axons. In 

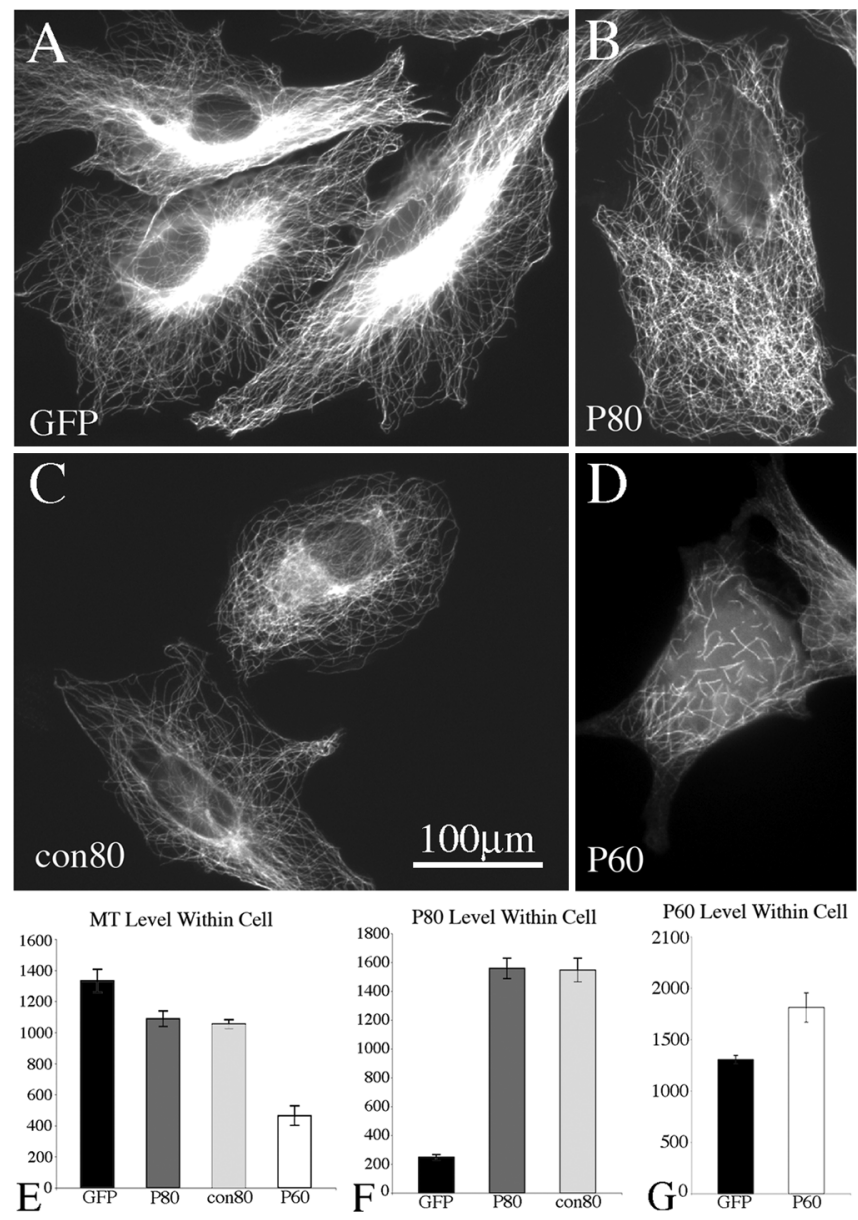

Figure 6. Effects of katanin constructs on microtubule levels in fibroblasts. $A-D$, Immunostaining of microtubules in RFL6 cells overexpressing EGFP, katanin P80, con80, or P60. E-G, Quantification of the data on microtubule levels and katanin (P80 and P60) levels. P80 and con 80 resulted in partial but not dramatic loss of microtubule mass (decreased by 18 and $16 \%$, respectively; $p<0.01)(\boldsymbol{B}, \boldsymbol{C}, \boldsymbol{E})$, even when expressed at high levels $(\sim 5$ times more than control; $p<0.001)(\boldsymbol{F}) . P 60$, in contrast, caused much greater severing and loss of microtubule mass (reduced by 63\%; $p<0.001$ ), even when expressed at much lower levels ( $28 \%$ increase; $p<0.01)$. Notably, P80 and con 80 , although not dramatically reducing microtubule levels, resulted in a loss of the tight concentration of microtubules in the centrosomal region $(\boldsymbol{B}, \boldsymbol{C})$. All of the microtubules in the cells overexpressing P60 are relatively short (a few micrometers in length), whereas the cells overexpressing P80 or con80 still display many long microtubules. Data ( $y$-axis) are expressed in AFUs. Scale bar, $100 \mu \mathrm{m}$.

contrast, there was a $20-23 \%$ loss of microtubules from dendrites as a result of expression of the P80 constructs, and there was a $44 \%$ diminution of microtubules in dendrites as a result of expression of the P60 construct. Thus, dendritic microtubules do not show the resistance to katanin displayed by microtubules in the axon. Quantitative data for stage 3 and stage 4 are shown in Figure $8, C c$ and $D d$, respectively. A more complete treatment of the statistical analyses is provided in the figure.

\section{Discussion}

Katanin is an abundant protein in the nervous system, the effects of which on microtubules can be very dramatic. Even modest experimental elevations in the levels of P60 katanin can cause significant depletion of microtubules, presumably via depolymerization after severing. The question arises as to how katanin is controlled, such that microtubules are not indiscriminately broken into tiny pieces and ultimately depolymerized throughout the neuron. Available evidence suggests that microtubule sever- ing is particularly noteworthy at the centrosome, at sites of branch formation, and within growth cones that begin to move again after having been stalled. In addition, microtubules need to be severed, albeit less avidly, throughout other compartments of the neuron to ensure that sufficient numbers of microtubules are able to undergo rapid transport. The precise relationship between microtubule length and transport remains unresolved but presumably requires a tight balance between short microtubules that move and long microtubules that act as railways for the movement (Hasaka et al., 2004). Microtubule severing also produces more free ends of microtubules, which are thought to be sites for the interaction of the microtubule with a number of other structures and proteins (Kornack and Giger, 2005). All of these observations are consistent with the idea that katanin-based microtubule severing is globally suppressed in the neuron, and that this suppression is relieved at key locales and phases of development.

In the present study, we quantitatively analyzed the levels of the two subunits in various tissues of the developing rat and found that the levels of P60 katanin uniformly exceed the levels of P80 katanin in all tissues and at all stages of development with the exception of the adult cortex. Moreover, we found that the ratio of the two subunits is dramatically different in the nervous system compared with other organs of the body. In the developing heart, for example, there are 10- to 20-fold lower levels of P80 compared with P60, whereas in the developing CNS, the ratio of P60:P80 is from $6: 1$ to $2: 1$. The fact that functional results are obtained when either one of the two subunits is overexpressed suggests a scenario whereby a molecule of $\mathrm{P} 60$ is functional without a corresponding molecule of P80, but that P60 is more potent when there is a corresponding P80 molecule available. These findings support the view that one level of control over microtubule severing in cells may be to regulate the ratio of the two katanin subunits. The fact that neurons contain very high levels of both subunits and a markedly lower ratio of P60/P80 compared with other tissues may be a clue to the manner by which katanin-induced severing of microtubules is orchestrated in the nervous system.

A previous study from our laboratory using cultured rat sympathetic neurons showed relatively uniform immunoreactivity throughout all neuronal compartments (Ahmad et al., 1999). These studies were performed with antibodies to human katanin, which we now believe were not optimal for these analyses. Using our newer antibodies, we have shown recently that there is some enrichment of $\mathrm{P} 60$ within the distal regions of growing axons (Karabay et al., 2004). Here, we pursued the issue in more detail using cultures of hippocampal neurons, which are particularly useful because they undergo a stereotyped sequence of developmental stages. Within the cell body, the levels of both P60 and P80 are essentially unaltered throughout the first three stages of development, after which the levels of both subunits increase. P60 then comes back down somewhat, but P80 continues to climb. P80 is far more concentrated in the cell body than P60. Within the processes, P80 levels are approximately the same in all types of processes except mature dendrites, in which the levels are approximately double the levels in any of the other processes at the various developmental stages. P60 and P80 are both just slightly higher at the tips of all types of processes compared with the processes themselves, except at early stage 4 , wherein both the tip of the axon and the dendrite show extremely dramatic concentrations of $\mathrm{P} 60$ but not P80. Also, there is a dramatic global increase in P60 levels during stage 4 but not in P80 levels. These observations, further supported by immunostaining on sections from intact mice, indicate that both the expression levels and the distribution of the 

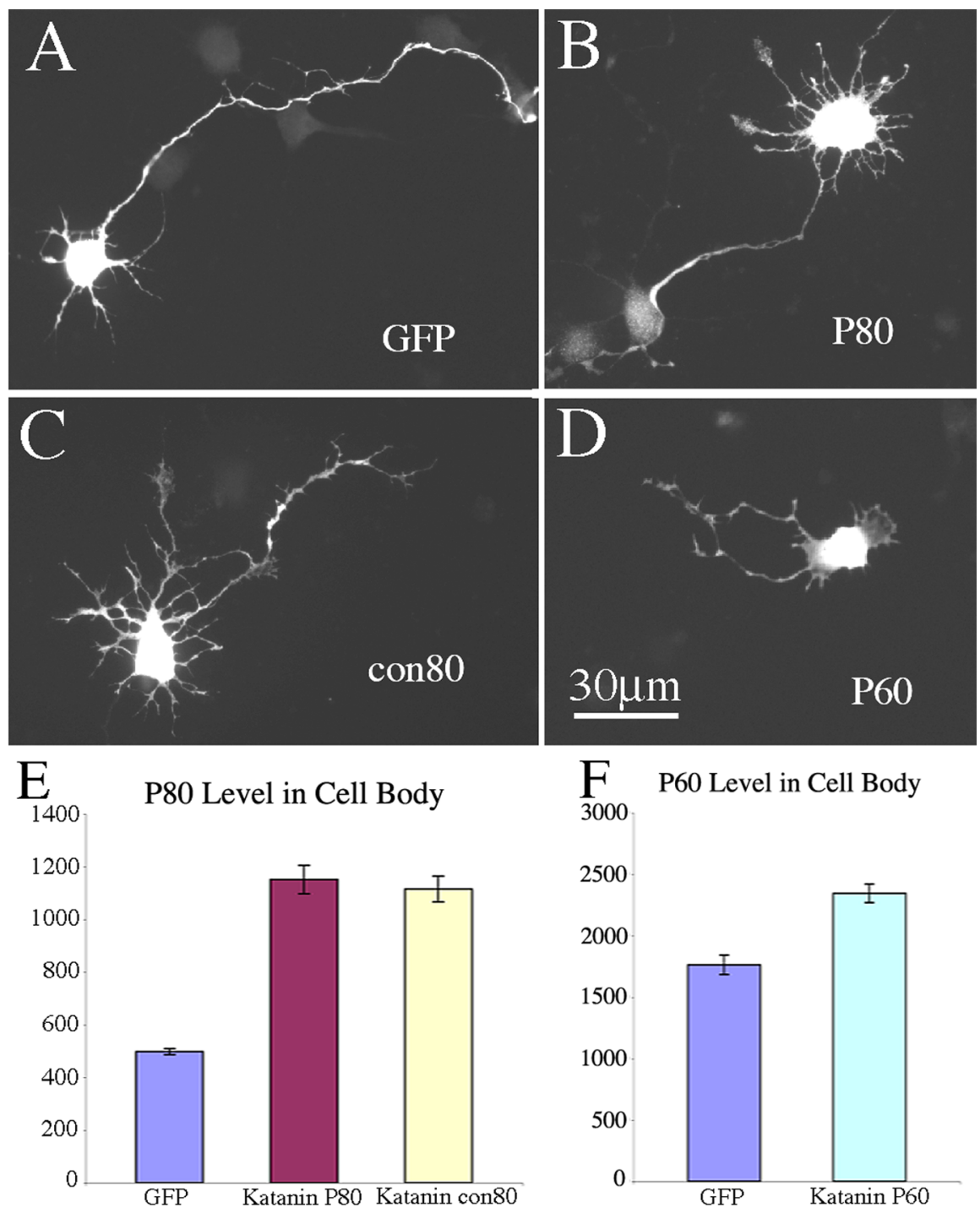

\section{G}

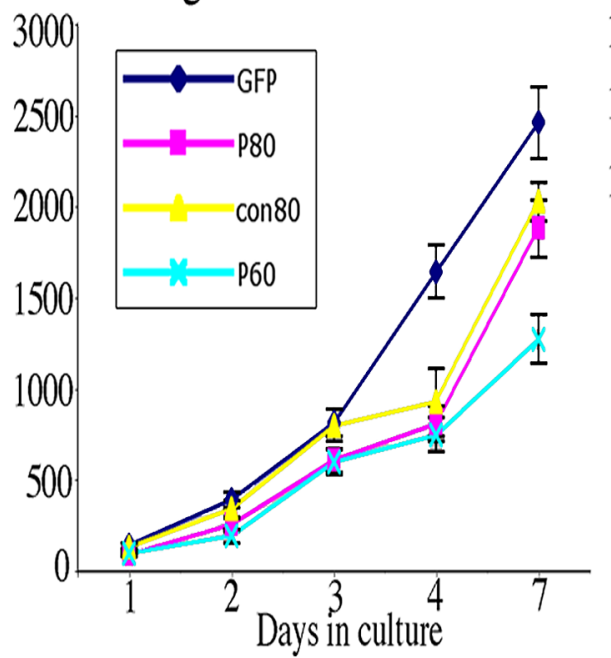

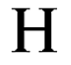
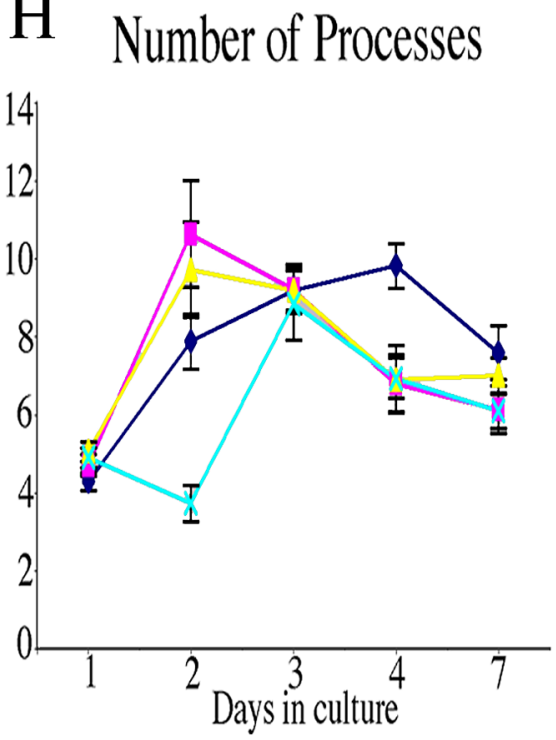

Figure 7. Quantitative analyses of process number and length on cultured hippocampal neurons at different developmental stages overexpressing katanin P80, con80, and P60. A-D, Immunostaining of EGFP for the cells that were transfected with the various constructs using EGFP as the tag. These cells were plated for $1 \mathrm{~d}$, transfected, and allowed to express for $1 \mathrm{~d}$. $\boldsymbol{E}-\boldsymbol{H}$, Quantification of data on the levels of katanin P80, con80, and P60 in the cell body, on process length, and on process number in hippocampal cultures at various days after plating, induced to express EGFP, P80-katanin, P60-katanin, or con80. The constructs

katanin subunits are differentially regulated during neuronal development.

The fact that $\mathrm{P} 80$ is present throughout all compartments of the neuron argues that its function cannot be solely to target katanin to the centrosome. Biochemical studies have established that P80 katanin consists of multiple domains with different functions. There is a domain that targets to the centrosome, a domain that augments microtubule severing by P60 katanin, and a third domain that appears to suppress microtubule severing (McNally et al., 2000). Our results using the full-length construct are consistent with the results of the biochemistry, which indicate that the net effect of the molecule is to augment microtubule severing. When we expressed a truncated version that only contains the domain that augments microtubule severing, the results were generally similar to those obtained with the fulllength molecule. However, the fact that P80 contains both domains suggests that there may be physiological mechanisms whereby each domain is used under different circumstances. For example, there may be local pathways for masking one or the other domain so that the net effect of P80 is to either assist or attenuate P60induced severing.

Our experiments expressing the katanin constructs indicate that various critical phases of neuronal development are more or less sensitive to alterations in katanin. Manipulation of katanin at key phases of development can produce markedly different results, depending on the severity of the effect on the microtubules. We found that process number is very sensitive to katanin manipulation around the time of axonal differentiation. Overexpression of $\mathrm{P} 60$ caused a diminution of process number, whereas overexpression of P80 caused an increase in process num-

\section{$\leftarrow$}

were expressed for only $1 \mathrm{~d}$ after various days in culture. Day 1 refers to cells that were transfected before plating. Note that day 2 represents a particularly sensitive developmental stage with regard to process number. The P60 construct diminishes process number, whereas the P80 constructs increase process number $(\boldsymbol{A}-\mathbf{D}, \boldsymbol{H})(p<0.05$ between control and $\mathrm{P} 80 /$ con $80 ; p<0.0001$ for control and $\mathrm{P} 60)$. With regard to the length of the processes, P80 and P60 overexpression decreased total process length by 30 and $50 \%$, respectively $(p<0.01)$. Day 4 is sensitive in terms of process number and length, with all constructs diminishing both $(p<0.01)$. The number of days in culture is shown on the $x$-axis. The $y$-axis shows the total length of all of the processes extended by the neuron in micrometers $(n>20)$. At all time points, overexpression of $\mathrm{P} 80$ and con 80 increases the protein level within the cell body by 2.3 times, whereas $P 60$ increases total levels by $\sim 33 \%(\boldsymbol{E}, \boldsymbol{F})$ ( $y$-axis is in AFUs). Scale bar, $30 \mu \mathrm{m}$. 

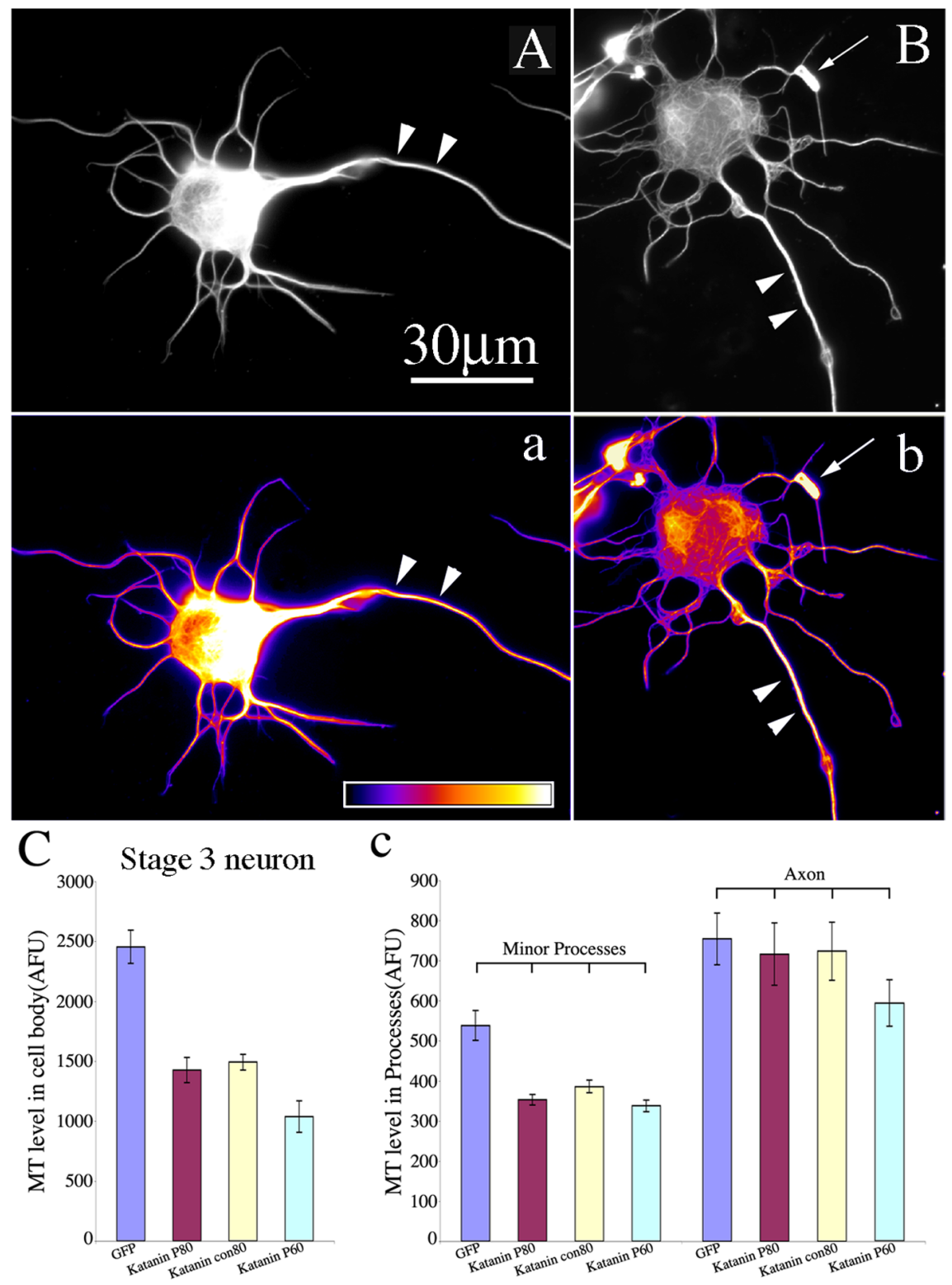

C
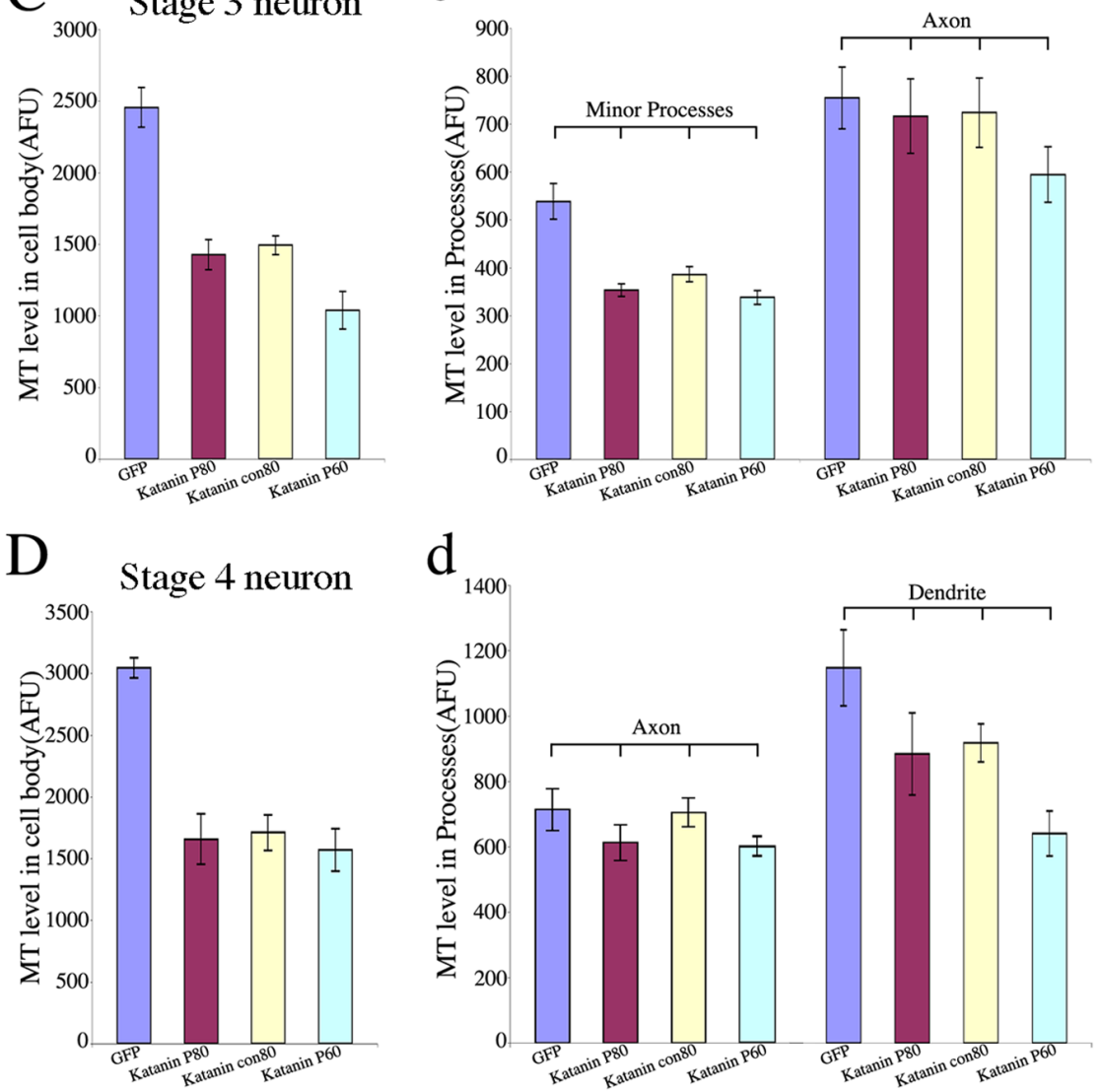

d

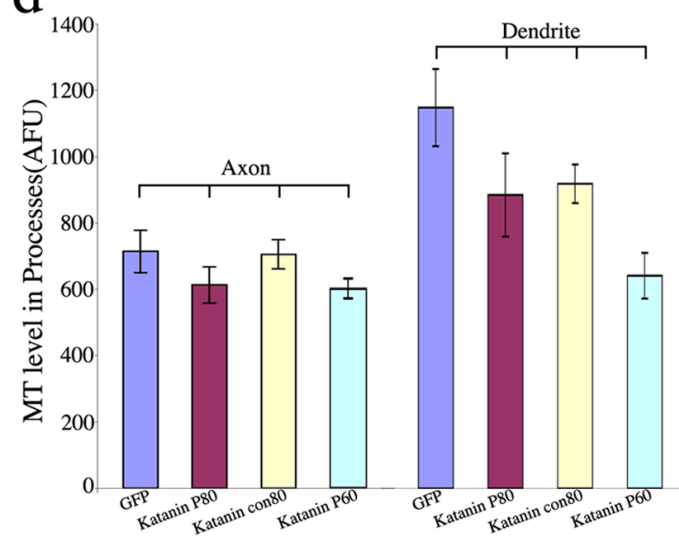

Figure 8. Quantitative analyses of microtubule levels in different regions of cultured hippocampal neurons overexpressing $\mathrm{P} 80$, con80, and P60. $\boldsymbol{A} \boldsymbol{a}$ and $\boldsymbol{B} \boldsymbol{b}$ are microtubule immunostainings of late stage 3 hippocampal neurons. $\boldsymbol{a}$ and $\boldsymbol{b}$ are the glow-scale pseudocolored images, with white indicating the highest level and purple indicating the lowest level (see color-coded bar). A $\boldsymbol{a}$ shows a control neuron expressing EGFP, and $\boldsymbol{B} \boldsymbol{b}$ shows a neuron overexpressing P60 katanin. Arrowheads point to the axon, and small arrows indicate twisted microtubules that are sometimes observed in neurons overexpressing P80, con 80 , or P60 (note also the bright structure in the top left of $\boldsymbol{B}$ and $\boldsymbol{D}$; (this is a small bundle of these contorted microtubules). Note that the axon ber. These results suggest that a mild increase in microtubule severing via P80 reconfigures the microtubule array in such a way as to promote the formation of more processes, presumably by enhancing release of microtubules from the centrosome or increasing the rate of exodus of microtubules from the cell body. Too much severing, as we observed in the past with P60 overexpression, produces a deleterious result by depleting too much microtubule mass (Karabay et al., 2004). Consistent with the spike in P60 levels observed during early dendritogenesis, this developmental stage is also particularly sensitive to experimental increases in either of the two katanin subunits, with regard to total process length. In no case did we observe an obvious effect on axonal branching, suggesting that augmentation of microtubule severing alone may not be sufficient to produce additional branches. It will be important to perform knock-down studies to ascertain whether diminishing the levels of either P60 or P80 has an effect on axonal branching; such studies are currently underway.

The fact that axonal microtubules are more resistant to overexpressed katanin than microtubules elsewhere in the neuron suggests that the mechanisms for attenuating katanin-based severing may be particularly pronounced or differentially regulated in the axon. We proposed that the fibrous microtubule-associated proteins (MAPs) within the neuron may protect the lattice of the microtubule from being accessed by katanin (Baas and Qiang, 2005). This finding is based on, in part, the

\section{$\longleftarrow$}

shows no diminution in fluorescence intensity as a result of P60 overexpression, whereas the cell body and immature ("minor") processes are dimmer. (c shows the quantification of microtubule mass in stage 3 neurons. Overexpression of the various katanin constructs in stage 3 neurons resulted in a $30-58 \%$ decrease in microtubule levels in cell bodies and minor processes $(p<0.01)$. In axons, overexpression of P80 or con80 did not cause any microtubule diminution (no significant difference; $p>0.05$ ), but overexpression of P60 caused a $20 \%$ loss of microtubules $(p<0.01)$. Dd shows the quantification of microtubule mass in stage 4 neurons. Overexpression of the various katanin constructs caused microtubule levels in the cell bodies to decrease by $44-50 \%$ ( $p<$ 0.01 ), which is similar to the results obtained from the cell bodies of stage 3 neurons. In the stage 4 axons, like stage 3 axons, P80 or con80 did not cause any microtubule diminution ( $p>0.05$ ). However, at stage 4 , there was also no diminution in microtubule levels in axons resulting from expression of the P60 construct $(p>0.05)$. In dendrites, there were 20 and $23 \%$ diminution in microtubule levels as a result of P80 and con80, respectively, and a $44 \%$ diminution in microtubule levels as a result of the P60 construct $(p<0.05$ ). Scale bar, $30 \mu \mathrm{m}$. 
observation that MAP4, a non-neuronal MAP, attenuates the severing of microtubules by katanin in vitro (McNally et al., 2002). Neurons in the brain do not express MAP4, but they abundantly express tau and MAP2, which share a similar microtubulebinding domain with MAP4. During neuronal development, the mature isoform of MAP2 becomes compartmentalized within cell bodies and dendrites, whereas the isoforms of tau that bind microtubules becomes compartmentalized into the axon (Dehmelt and Halpain, 2004). The fact that axonal microtubules are more resistant to overexpressed katanin suggests that tau may be enriched in the axon to afford strong protection against potential severing, and that tau may be a better protector than MAP2. The MAP-protection model is attractive, because the association of fibrous MAPs with microtubules is regulated by phosphorylation events (Avila et al., 1994; Ebneth et al., 1999), which can be influenced by signaling cascades relevant to growth cone motility and branch formation. Consistent with this possibility, biochemical studies on katanin have established that its severing properties are regulated in part by phosphorylation of other proteins (Vale, 1991; McNally et al., 2002). Thus, it may be that MAPs such as tau protect the microtubules against severing until a signaling event results in the dissociation of MAPs from the microtubules in a particular locale of the neuron, such as a site of impending branch formation.

We acknowledge that neurons also express spastin, a protein with mutations that give rise to spastic paraplegia in humans. Spastin has been shown to have microtubule-severing properties comparable with P60 katanin (Errico et al., 2002; Evans et al., 2005), and hence it will be of interest to investigate how the activities of katanin and spastin are coordinated in the neuron.

\section{References}

Ahmad FJ, Yu W, McNally FJ, Baas PW (1999) An essential role for katanin in severing microtubules in the neuron. J Cell Biol 145:305-315.

Avila J, Dominguez J, Diaz-Nido J (1994) Regulation of microtubule dynamics by microtubule-associated protein expression and phosphorylation during neuronal development. Int J Dev Biol 38:13-25.

Baas PW, Buster DW (2004) Slow axonal transport and the genesis of neuronal morphology. J Neurobiol 58:3-17.

Baas PW, Qiang L (2005) Neuronal microtubules: when the MAP is the roadblock. Trends Cell Biol 15:183-187.

Buster DW, Baird DH, Yu W, Solowska JM, Chauviere M, Mazurek A, Kress M, Baas PW (2003) Expression of the mitotic kinesin Kif15 in postmitotic neurons: implications for neuronal migration and development. J Neurocytol 32:79-96.

Dehmelt L, Halpain S (2004) The MAP2/tau family of microtubuleassociated proteins. Genome Biol 6:204.

Dent EW, Callaway JL, Szebenyi G, Baas PW, Kalil K (1999) Reorganization and movement of microtubules in growth cones and developing interstitial branches. J Neurosci 9:8894-8904.

Dotti CG, Sullivan CA, Banker GA (1988) The establishment of polarity by hippocampal neurons in culture. J Neurosci 8:1454-1468.

Ebneth A, Drewes G, Mandelkow EM, Mandelkow E (1999) Phosphorylation of MAP2c and MAP4 by MARK kinases leads to the destabilization of microtubules in cells. Cell Motil Cytoskeleton 44:209-224.

Errico A, Ballabio A, Rugarli EI (2002) Spastin, the protein mutated in autosomal dominant hereditary spastic paraplegia, is involved in microtubule dynamics. Hum Mol Genet 15:153-163.

Evans KJ, Gomes ER, Reisenweber SM, Gundersen GG, Lauring BP (2005) Linking axonal degeneration to microtubule remodeling by Spastinmediated microtubule severing. J Cell Biol 14:599-606.

Hartman JJ, Mahr J, McNally K, Okawa K, Iwamatsu A, Thomas S, Cheesman J, Heuser J, Vale RD, McNally FJ (1998) Katanin, a microtubulesevering protein, is a novel AAA ATPase that targets to the centrosome using a WD40-containing subunit. Cell 93:277-287.

Hasaka TP, Myers KA, Baas PW (2004) Role of actin filaments in the axonal transport of microtubules. J Neurosci 24:11291-11301.

Karabay A, Yu W, Solowska JM, Baird DH, Baas PW (2004) Axonal growth is sensitive to the levels of katanin, a protein that severs microtubules. J Neurosci 24:5778-5788.

Kornack DR, Giger RJ (2005) Probing microtubule +TIPs: regulation of axon branching. Curr Opin Neurobiol 15:58-66.

McNally FJ, Vale RD (1993) Identification of katanin, an ATPase that severs and disassembles stable microtubules. Cell 75:419-429.

McNally KP, Bazirgan OA, McNally FJ (2000) Two domains of p80 katanin regulate microtubule severing and spindle pole targeting by $\mathrm{P} 60$ katanin. J Cell Sci 113:1623-1633.

McNally KP, Buster D, McNally FJ (2002) Katanin-mediated microtubule severing can be regulated by multiple mechanisms. Cell Motil Cytoskeleton 53:337-349.

Quarmby LM, Lohret TA (1999) Microtubule severing. Cell Motil Cytoskeleton 43:1-9.

Sambrook J, Fritsch EF, Maniatis T (1989) Molecular cloning. A laboratory manual, Ed 2, pp 18.47-18.71. Cold Spring Harbor, NY: Cold Spring Harbor Laboratory.

Solowska JM, Mazurek A, Weinberger L, Baird DH (2002) Pontocerebellar axon guidance: neuropilin-1 and Sema3A-sensitivity gradients across basilar pontine nuclei, Sema3A variation across cerebellum. Mol Cell Neurosci 21:266-284.

Vale RD (1991) Severing of stable microtubules by a mitotically activated protein in Xenopus egg extracts. Cell 64:827-839.

Wang L, Brown A (2002) Rapid movement of microtubules in axons. Curr Biol 12:1496-1501.

Yu W, Baas PW (1994) Changes in microtubule number and length during axon differentiation. J Neurosci 14:2818-2829.

Yu W, Centonze VE, Ahmad FJ, Baas PW (1993) Microtubule nucleation and release from the neuronal centrosome. J Cell Biol 122:349-359.

Yu W, Ahmad FJ, Baas PW (1994) Microtubule fragmentation and partitioning in the axon during collateral branch formation. J Neurosci 14: 5872-5884. 